Journal of Patient-Centered

$1-28-2019$

\title{
Acknowledgment of 2018 JPCRR Peer Reviewers
}

Follow this and additional works at: https://aah.org/jpcrr

Part of the Health Services Research Commons, and the Medical Humanities Commons

\section{Recommended Citation}

Acknowledgment of 2018 JPCRR peer reviewers. J Patient Cent Res Rev. 2019;6:51.

Published quarterly by Midwest-based health system Advocate Aurora Health and indexed in PubMed Central, the Journal of Patient-Centered Research and Reviews (JPCRR) is an open access, peer-reviewed medical journal focused on disseminating scholarly works devoted to improving patient-centered care practices, health outcomes, and the patient experience. 


\section{Acknowledgment of 2018 JPCRR Peer Reviewers}

The editors of Journal of Patient-Centered Research and Reviews sincerely appreciate all submission reviewers from the past calendar year. Quality assurance of the content published within the journal is largely dependent on the volunteer contributions of these individual experts.

Aboud Affi

Betty Amuzu

Andy Anderson

Deanna Attai

Diane Austin

Dennis Baumgardner

Lisa Berger

Jacob Bidwell

Lora Black

John Brill

Al Bugazia

Christine Casselman

Ron Cisler

Jacqueline Coby-Beaver

Roberta Coles

David Dhanraj

Elizabeth Dickson Michelson

Nicole Eull

Julien Fahed

Michael Farrell

Jennifer Fink

John Frey, III
Gina Green-Harris

Jennifer Hartlaub

Kayla Heslin

Julian Hong

Mark Kaeppler

Carla Kelly

Edward Kim

Christopher Klink

Marianne Klumph

Kjersti Knox

Fabiana Kotovicz

Jessica Kram

Marianna LaNoue

Melissa Lemke

Jacob Lescher

Nicole Lohr

Regina Manansala

Michelle Maternowski

Susan McRoy

Alyssa Mohorek

Bruce Morgenstern

Chris Morley
George Morris, III

Donald Nease, Jr.

David Nelson

Kushal Patel

Becky Pogacar

Hershel Raff

Katharine Rendle

Mark Robinson

Steve Rommelfanger

MaryAnne Scherer

Nina Shah

Robyn Shapiro

Deborah Simpson

Michelle Simpson

Maureen Smith

Lisa Sullivan Vedder

Heather Tick

Michael Thompson

Jackie Tillett

Mindy Waite 